\title{
Can the Physics of Time Be Helpful in Solving the Differential Eigenequations Characteristic for the Quantum Theory?
}

\author{
Stanisław Olszewski \\ Institute of Physical Chemistry, Polish Academy of Sciences, Warsaw, Poland \\ Email: olsz@ichf.edu.pl
}

How to cite this paper: Olszewski, S. (2019) Can the Physics of Time Be Helpful in Solving the Differential Eigenequations Characteristic for the Quantum Theory? Advances in Pure Mathematics, 9, 228-241. https://doi.org/10.4236/apm.2019.93011

Received: January 8, 2019

Accepted: March 26, 2019

Published: March 29, 2019

Copyright $\odot 2019$ by author(s) and Scientific Research Publishing Inc. This work is licensed under the Creative Commons Attribution International License (CC BY 4.0).

http://creativecommons.org/licenses/by/4.0/

\section{(c) (i) Open Access}

\begin{abstract}
The main differential equations of quantum theory are the eigenequations based on the energy operator; they have the energy as eigenvalues and the wave functions as eigenfunctions. A usual complexity of these equations makes their accurate solutions accessible easily only for very few physical cases. One of the methods giving the approximate solutions is the Schrödinger perturbation theory in which both the energies and wave functions of a more complicated eigenproblem are approached with the aid of similar parameters characteristic for a less complicated eigenproblem. No time parameter is necessary to be involved in these calculations. The present paper shows that the Schrödinger perturbation method for non-degenerate stationary quantum states, i.e. the states being independent of time, can be substantially simplified by applying a circular scale of time separately for each order of the perturbation theory. The arrangement of the time points on the scale, combined with the points contractions, gives almost immediately the series of terms necessary to express the stationary perturbation energy of a given eigenproblem. The Schrödinger's method is compared with the Born-Heisenberg-Jordan perturbation approach.
\end{abstract}

\section{Keywords}

Differential Eigenequations in Quantum Theory, Perturbation Method, Circular Scale of Time

\section{Introduction}

There exists a multitude of the mathematical methods which help the people in science, engineering and everyday life. The aim of the present paper is to demonstrate a reversed example, namely that physical ideas on time can be helpful in 
the treatment of the mathematics connected with solving of the differential eigenequations.

In general a differential equation is represented by a mutual dependence between some unknown function, its derivative or derivatives, and one or more independent variables. Evidently the mathematics has its methods to solve such equations. In some special cases the solutions can be analytic in their form, but in general there exist the numerical methods which can provide us systematically with the required results.

In the modern non-relativistic quantum theory, began by Schrödinger in the middle of 1920's, the differential eigenequations played an important role [1] [2] [3] [4]. This comes from the postulate that the stationary states of the energy of a physical system, say an atom or an atomic ensemble, should be given by the eigenvalues of the Hamiltonian energy operator composed usually from the kinetic differential part and a multiplicative part acting together on the wave function representing the examined electron system. In effect the operators of a mixed character-partly differential and partly multiplicative-can enter into play giving usually a complicated mathematical problem.

In case of the presence of many electrons the resulted eigenproblem can be much too complicated to be attacked with the aid of conventional methods applied to the differential equations. An example can be the accurate differential equation describing the electron wave function in a metal. The equation should be necessarily transformed into a more simple form due to an analysis of the physical conditions governing the behaviour of electrons near the nuclei of the metal atoms on the one side, and in the areas rather distant from the nuclei on the other side; see e.g. [5]. The effect of a simplification of such kind can be the equations which occur to be satisfactory in calculating many of the physical properties concerning metals. But this is only one advantage due to the use of a simplified solution. Another advantage-raised especially by Wigner-is that a successful simplification of the necessary mathematical equations allows us for a better insight and understanding of the physics entering the problem [6]. Such insight can be easily smoothed out by a powerful computer whose task was to present only the effective numerical data for the physical parameters characterizing the examined piece of matter, but the calculations do not give a look into the play of forces which are responsible for the observed phenomena and results.

In the present paper we discuss the use of a specific time notion and time scale suitable in solving a kind of the differential eigenequations entering the quantum mechanics.

\section{Schrödinger's Quantum Mechanics of the Unperturbed and Perturbed Differential Eigenequations}

A crucial mathematical difficulty of the quantum theory, i.e. the complexity of the eigenquations, became evident already for Schrödinger himself [3]. In fact only very special, and mathematically rather simple, objects like the harmonic 
oscillator or hydrogen atom, could be successfully attacked by the quantum theory from before of 1925. Already the spectrum of the helium atom, the next atom in the periodic table after the hydrogen, could not be presented by that theory. However, by the use of the idea of the de Broglie waves of matter, Schrödinger could formulate his second-order differential equations suitable to be applied to the electron spectrum of any atom. Moreover, the appropriate Schrödinger equations, called sometimes the wave-mechanical equations, could be applied to molecules and solids. In practice the equations could be easily founded-but not solved-for any multi-electron problem because of our knowledge of the electrostatic forces which govern any many-electron system: The forces dictated by the Coulomb law act between the electrons and atomic nuclei, as well as-separately-between the purely multi-electron and many-nuclear ensembles.

The idea of construction of the differential eigenequation suitable for each case was based on the classical Hamilton expression for the energy possessed separately by any particle entering the system increased by the interaction energy between different particles. For example any single electron particle, say in an atom, has its kinetic energy represented by the expression ( $m$ is the electron mass):

$$
E_{\text {kin }}=\frac{1}{2 m}\left(p_{x}^{2}+p_{y}^{2}+p_{z}^{2}\right),
$$

where $p_{x}, p_{y}$ and $p_{z}$ are the Cartesian coordinates of the particle momentum, increased by the potential energy due to the electrostatic interaction between an electron and the atomic nucleus:

$$
E_{\mathrm{pot}}=-\frac{Z e^{2}}{r_{i}} .
$$

Here $r_{i}$ is a distance between the ith electron having charge $-e$ and the nucleus having $Z e$ as its electrostatic charge.

The collective electrostatic energy between the electrons is represented by the Coulomb interaction energy term equal to

$$
E_{\text {int }}=\frac{1}{2} \sum_{\substack{i \\ i \neq j}} \sum_{\substack{j \\ r_{i j}}}
$$

where $r_{i j}$ is a distance between the electrons $i$ and $j$.

Usually the nucleus is assumed to be at rest, so its kinetic energy is neglected. In effect the total classical Hamiltonian for an atom is a sum of (1), (2) and (3) extended respectively over the number of the electron particles. An exclusion of the self-interaction energy of electrons should be done by eliminating the terms corresponding to $i=j$ from the sum entering (3).

The next step-suggested by the de Broglie waves-was to change $p_{x}, p_{y}$ and $p_{z}$ in (1) into the differential operators:

$$
p_{x} \rightarrow-i \frac{\partial}{\partial x}=\hat{p}_{x}, \quad p_{y} \rightarrow-i \frac{\partial}{\partial y}=\hat{p}_{y}, \quad p_{z} \rightarrow-i \frac{\partial}{\partial z}=\hat{p}_{z} .
$$


The Planck constant $\mathrm{h}$ divided by $2 \pi$ is usually entering (4); in the present case this constant term is put equal to 1 .

Therefore instead of the classical $E_{\text {kin }}$ in (1) we obtain the kinetic-energy operator

$$
\hat{E}_{\text {kin }}=\frac{1}{2 m}\left(\hat{p}_{x}^{2}+\hat{p}_{y}^{2}+\hat{p}_{z}^{2}\right)=-\frac{1}{2 m}\left(\frac{\partial^{2}}{\partial x^{2}}+\frac{\partial^{2}}{\partial y^{2}}+\frac{\partial^{2}}{\partial z^{2}}\right)=-\frac{1}{2 m} \nabla^{2} .
$$

The operators $E_{\mathrm{pot}}$ and $E_{\text {int }}$-because they depend solely on the electron position-remain of a multiplicative character given in (2) and (3), therefore

$$
\begin{gathered}
\hat{E}_{\text {pot }}=E_{\text {pot }}, \\
\hat{E}_{\text {int }}=E_{\text {int }} .
\end{gathered}
$$

In effect the quantum problem of the atomic electron energy postulated to be solved becomes

$$
\hat{H} \psi=\hat{E}_{\mathrm{tot}} \psi=\left[\sum_{i}\left(\hat{E}_{\mathrm{kin}}^{(i)}+\hat{E}_{\mathrm{pot}}^{(i)}\right)+\hat{E}_{\mathrm{int}}\right] \psi=E_{\mathrm{tot}} \psi,
$$

or more briefly

$$
\hat{E}_{\text {tot }} \psi=E_{\text {tot }} \psi,
$$

where $E_{\text {tot }}$ on the right of (9) is a number. The sum in (8) is extended over the number of electron particles labelled by $i$.

Because of

$$
\hat{E}_{\text {kin }} \neq E_{\text {kin }}
$$

the problem becomes mathematically a differential eigenequation for the electron wave functions

$$
\begin{aligned}
& \psi_{\alpha}\left(x_{1}, y_{1}, z_{1}, x_{2}, y_{2}, z_{2}, x_{3}, y_{3}, z_{3}, \cdots\right), \\
& \psi_{\beta}\left(x_{1}, y_{1}, z_{1}, x_{2}, y_{2}, z_{2}, x_{3}, y_{3}, z_{3}, \cdots\right), \\
& \psi_{\gamma}\left(x_{1}, y_{1}, z_{1}, x_{2}, y_{2}, z_{2}, x_{3}, y_{3}, z_{3}, \cdots\right),
\end{aligned}
$$

and electron eigenenergies

$$
E_{\text {tot }}=E_{\text {tot }}^{(\alpha)}, E_{\text {tot }}^{(\beta)}, E_{\text {tot }}^{(\gamma)}, \cdots
$$

Because of the equality of the operators

$$
\hat{E}_{\text {tot }}=\hat{H}
$$

the operator $\hat{H}$ in (13) is called the Hamiltonian, or energy operator.

\section{Classification of the Wave Functions and Energies into Non-Degenerate and Degenerate States}

Any wave function

$$
\psi=\psi\left(x_{1}, y_{1}, z_{1}, x_{2}, y_{2}, z_{2}, \cdots\right)
$$

defines some electron distribution called the electron density

$$
\varrho=\psi^{*} \psi \text {. }
$$


In general there can exist an infinite number of solutions $\psi$ and $E_{\text {tot }}$ of the eigenequation (9):

$$
\psi=\psi_{\alpha}, \psi_{\beta}, \psi_{\gamma}, \cdots
$$

(see (11)) and

$$
E_{\text {tot }}
$$

given in (12). In case when all eigenvalues (12) are different, the eigenproblem is called non-degenerate, but in case some of solutions (12) are found to be equal, the eigenproblem is called degenerate. Examples of the solutions (16) and (17) are given in [7] and [8].

\section{Difficulty in Solving the Schrödinger Equations and Application of the Perturbation Method}

The problem, noticed already by Schrödinger [3], is that the solution of his equation (8)-(9) becomes, in general, an extremely complicated task. Only very few physical systems provide us with simple expressions for $\psi$ and $E_{\text {tot }}$. In reply to this difficulty Schrödinger proposed to solve many eigenproblems by considering them similar to the solved problems in the sense that the operator

$$
\hat{E}_{\text {pot }}^{\text {(unsolved) }}-\hat{E}_{\text {pot }}^{\text {(solved) }}=\hat{V}^{\text {per }}
$$

is of a small size and, for the sake of convenience, dependent solely on the position vector, or vectors, of the kind of $\boldsymbol{r}$. Therefore, for a single set of the Cartesian coordinates $x, y, z$, we have

$$
\hat{V}^{\mathrm{p} e r}=V^{\mathrm{p} e r}(\vec{r}),
$$

so $V^{\text {per }}$ remains of a multiplicative character. The essence of the perturbation method becomes to calculate $E_{\mathrm{tot}}^{\text {per }}$ and $\psi^{\text {per }}$-the perturbed eigenenergies and eigenfunctions due to the eigenproblem

$$
\left(\hat{H}+\lambda V^{\mathrm{per}}\right) \psi^{\mathrm{per}}=E_{\mathrm{tot}}^{\mathrm{per}} \psi^{\mathrm{per}}
$$

with the aid of $E_{\text {tot }}$ and $\psi$ obtained due to solving the unperturbed problem. The $\lambda$ entering (20) is assumed to be a small number. It makes that for $\lambda=0$ we have the unperturbed problem, whereas $\lambda=1$ gives a full perturbed problem to be solved.

If we focus our attention only on the energy-which is usually a more seeked parameter than the wave function-we obtain

$$
E_{\mathrm{tot}}^{\mathrm{per}}=E_{\mathrm{tot}}+\lambda \Delta E_{1}+\lambda^{2} \Delta E_{2}+\cdots .
$$

For $\lambda=0$ the first term on the right of (21) is equal to one of the unperturbed energies in (12). The other terms, labelled by

$$
\lambda \Delta E_{1}, \lambda^{2} \Delta E_{2}, \lambda^{3} \Delta E_{3}, \cdots
$$

are for $\lambda=1$ equal to the full perturbation corrections of some unperturbed energy $E_{\text {tot }}^{(i)}$ belonging respectively to the perturbation orders:

$$
N=1, N=2, N=3, \cdots .
$$


In general the corrections (22) can be constructed from the matrix elements

$$
\left\langle\psi_{\alpha}\left|V^{\mathrm{per}}\right| \psi_{\beta}\right\rangle
$$

where $\psi_{\alpha}$ and $\psi_{\beta}$ are the unperturbed wave functions, and from the unperturbed energy differences

$$
E_{\text {tot }}^{(i)}-E_{\text {tot }}^{(\alpha)}, E_{\text {tot }}^{(i)}-E_{\text {tot }}^{(\beta)}, E_{\text {tot }}^{(i)}-E_{\text {tot }}^{(\gamma)}, \cdots
$$

where evidently

$$
\alpha, \beta, \gamma, \cdots \neq i
$$

if the unperturbed energy state $E_{\text {tot }}^{(i)}$ is taken into the perturbation process.

According to [9] and [10] any $\Delta E_{N}$ is composed of $S_{N}$ different kinds of the perturbation terms built up of (24) and (25). Excepting for the case of $N=1$, any of the $S_{N}$ terms

$$
S_{N}=\frac{(2 N-2) !}{N !(N-1) !}
$$

taken for $N>1$ is represented by an infinite sum of the expressions containing (24) and (25). For example for $N=2$ we have a single perturbation term because

$$
S_{2}=\frac{(2 \times 2-2) !}{2 ! 1 !}=1
$$

which has the form

$$
\Delta E_{2}=\sum_{j \neq i} \frac{\left\langle i\left|V^{\text {per }}\right| j\right\rangle\left\langle j\left|V^{\text {per }}\right| i\right\rangle}{E^{(i)}-E^{(j)}} .
$$

It should be noted that $S_{N}$ can attain large numbers already for rather low $N$ :

$$
S_{1}=1, S_{2}=1, S_{3}=2, S_{4}=5, S_{5}=14, S_{6}=42, S_{7}=132, S_{8}=429, \cdots
$$

But for example for $N=20$ we have

$$
S_{20} \cong 1.767 \times 10^{9} \text {. }
$$

\section{Construction of the Energy Perturbation Terms $\Delta E_{N}$ Belonging to Different $N$ and the Problem of Time}

Schrödinger did not use the notion of time in solving his perturbation eigenequations [3]. In principle the unperturbed and perturbed Schrödinger eigenequations did not contain the time as an independent variable. Also in course of solving his eigenequations he followed a purely mathematical procedure. But time was included in the perturbation process for energy, and corresponding diagrams, by Feynman [11] [12]. By using the scale of time known from the everyday experience, the time parameter $t$ can be extended from the minus to plus infinity, viz.

$$
-\infty<t<\infty .
$$

In this case, it became possible to calculate the $S_{N}$ perturbation terms belonging to a given order $N$ with the aid of 


$$
P_{N}=(N-1) !
$$

terms corresponding to the Feynman diagrams. For large $N$ the number $P_{N}$ heavily exceeds $S_{N}$. For example for $N=20$ we have

$$
P_{20}=19 ! \cong 1.216 \times 10^{17}
$$

which makes the ratio

$$
P_{20} / S_{20}=(1.216 / 1.767) \times 10^{8}
$$

equal to a very large number. In effect the calculation of numerous individual $S_{20}$ terms done with the aid of the $P_{20}$ terms can be a very complicated task.

But an evident simplification of the access to the Schrödinger perturbation terms can be afforded by a suitable choice of the scale of time which labels the collisions of a quantum system with the perturbation potential $V^{\text {per }}$ [13]-[18]. The scale is assumed to be of a circular character remaining similar for any $N$. This means that for any $N$ points of time considered on the scale, only $N-1$ points of time, viz.

$$
1,2,3,4, \cdots, N-1,
$$

are active in the collision done on a circular way with $V^{\text {per }}$. The $N$ th point is considered to be a beginning-end point of the scale and has no participation in the collisions process.

But a travel of a quantum system along the set of points given in (34) gives only one of the $S_{N}$ terms of the perturbation energy belonging to $N$. Such situation, when $S_{N}$ is reduced to a single term, holds for the case of

$$
S_{1}=1(N=1) \text {. }
$$

The time scale corresponding to $N=1$ is given in Figure 1. Evidently the scale possesses solely a single time point which is the beginning-end point of the scale.

For $N=2$ we have a situation represented by Figure 2. Here again

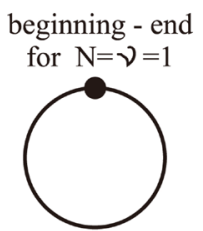

Figure 1. Time scale for the perturbation order $N=1$. Only one time point-the beginning-end point of the scale-is indicated.

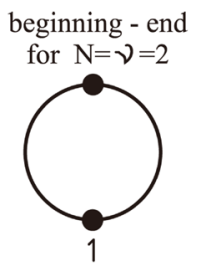

Figure 2. Time scale for the perturbation order $N=2$. Beyond of the beginning-end point of the scale, only point 1 of collision with the perturbation potential $V$ is indicated. 


$$
S_{2}=S_{1} \times S_{1}=1
$$

gives a single diagram, but beyond of the beginning-end point we have still one time point 1 present on the scale.

The energy term due to Figure 1 is

$$
\Delta E_{1}=\left\langle i\left|V^{\mathrm{per}}\right| i\right\rangle \equiv\langle V\rangle
$$

assuming that the energy of an unperturbed state $i$ is perturbed, whereas the perturbed energy term due to Figure 2 is that given in (29). An abbreviated formula for $\Delta E_{2}$ is:

$$
\Delta E_{2}=\langle V P V\rangle
$$

since

$$
P=\frac{1}{E^{(i)}-E^{(j)}} .
$$

The presence of a single $P$ implies a single summation within the expression (38) over index $j$, evidently with exclusion of the index $i$; see (29).

For $N>2$ we have $S_{N}>1$; see (30). The remaining terms $S_{N}$ are obtained from contractions of the time points which are free on the circular scale; see Section 6.

\section{Time-Point Contractions on a Circular Scale and Their Contribution to the Perturbation Energy}

We limit our further calculations to the orders $N=3,4$ and 5, giving respectively the number of the perturbation terms equal to

$$
S_{3}=2, S_{4}=5
$$

and

$$
S_{5}=14
$$

see (30). The number of free points of time remaining on the scale is respectively

$$
3-1=2,4-1=3,5-1=4
$$

for $N=3,4$ and 5 .

The points (42), when they are remaining free, are arranged successively on the main loop of time characteristic for a given $N$. The main loop of time means that it contains the beginning-end point of the time scale. When no time-point contractions on the main loop are present, the perturbation terms due to the loop-in the notation presented earlier in Section 5 for $N=1$ and $N=2$ -are:

$$
\langle V P V P V\rangle
$$

for the loop corresponding to $N=3$,

$$
\langle V P V P V P V\rangle
$$

for the loop corresponding to $N=4$, and 


$$
\langle V P V P V P V P V\rangle
$$

for the loop corresponding to $N=5$. The loop corresponding to $N=3$ is presented in Figure 3.

We see that the number of symbols $P$ entering the formulae (43)-(45) is equal to $N-1$, whereas the number of $V$ is equal to $N$.

In the next step the contractions of the time points whose number is given in (42) should be taken into account. The contractions provide us with the side loops touching the main loop of time. In the first case of (42) $(N=3)$ we have only two free points of time labelled by 1 and 2 . They can give only a single contraction

$$
1: 2
$$

the diagram connected with contraction in (46) is given in Figure 4. We see that the side loop due to (46) is identical with the loop of time characteristic for $N=1$. Therefore the energy contribution due to contraction (46) should be the term

$$
\left\langle i\left|V^{\mathrm{per}}\right| i\right\rangle=\langle V\rangle .
$$

But the merging of the time points given in (46) provides us with the merging of two $P$ separated by $V$ in (43). In effect the contribution to the perturbation energy due to (46) is represented by the formula

$$
\left\langle V P^{2} V\right\rangle
$$

A general rule is that the total number of $V$ and $P$ in the energy formulae belonging to a given $N$ does not change, so for $N=3$ it should contain three terms $V$ and two terms $P$; see (43). This implies that the total second perturbation term for $N=3$ is

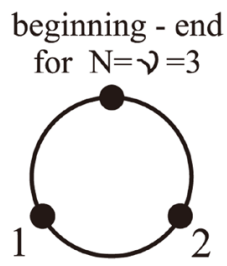

Figure 3. Time scale for the perturbation order $N=3$ having no contraction of the free time points 1 and 2.

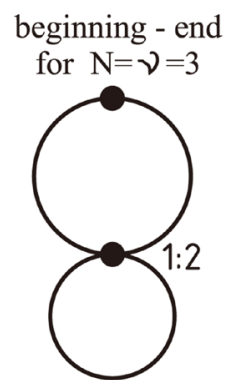

Figure 4. Time scale for the perturbation order $N=3$ having the contraction point 1:2. 


$$
-\langle V\rangle\left\langle V P^{2} V\right\rangle=-\Delta E_{1}\left\langle V P^{2} V\right\rangle .
$$

The minus sign in front of (49) is dictated by the fact that an even number of terms enter the product representing the whole term (49). In case we have an odd number of terms entering the product, the sign before the whole perturbation term should be positive.

It is easy to study the contractions of the free time points presented in (42) for $N=4$ (in this Section) and $N=5$ (in Section 7).

The three time points (1,2 and 3) present in the case of $N=4$ give four kinds of contractions:

$$
1: 2,1: 3,2: 3 \text { and } 1: 2: 3
$$

Together with the formula (44) the contractions in (50) give

$$
S_{4}=5
$$

perturbation terms belonging to $N=4$. We obtain

$$
\begin{gathered}
1: 2 \rightarrow-\left\langle V P^{2} V P V\right\rangle\langle V\rangle=-\left\langle V P^{2} V P V\right\rangle \Delta E_{1}, \\
1: 3 \rightarrow-\left\langle V P^{2} V\right\rangle\langle V P V\rangle=-\left\langle V P^{2} V\right\rangle \Delta E_{2},
\end{gathered}
$$

because the side loop of time due to contraction $1: 3$ has a free point 2 on it which makes the loop identical to that characteristic for $\Delta E_{2}$,

$$
2: 3 \rightarrow-\left\langle V P V P^{2} V\right\rangle\langle V\rangle=-\left\langle V P V P^{2} V\right\rangle \Delta E_{1}
$$

is symmetrical to (52), and

$$
1: 2: 3 \rightarrow\left\langle V P^{2} V\right\rangle\langle V\rangle\langle V\rangle=\left\langle V P^{2} V\right\rangle\left(\Delta E_{1}\right)^{2},
$$

because three points of time merge together and they give two side loops of time each representing the energy contribution characteristic for $N=1$. A general rule for contractions of the time points is that the loops created by them should not cross; see e.g. [13].

\section{Time Points, Their Contractions and the Perturbation Energy Terms Belonging to the $\operatorname{Order} N=5$}

The time points being free on the main loop of time representing the perturbation order $N=5$ are

$$
1,2,3 \text {, and } 4 \text {. }
$$

(Point 5 becomes the beginning-end point of time on the loop.) The energy term due to the free time points in (56) is presented in the formula (45). But since for $N=5$ we have the number

$$
S_{5}=14
$$

of the Schrödinger perturbation terms, the remainder of $14-1=13$ terms should be calculated. They come respectively from the following contractions of the time points in (56):

$$
1: 4,1: 2: 4,1: 3: 4,1: 2: 3: 4
$$




$$
2: 4,2: 3: 4
$$$$
3: 4 \text {. }
$$

In case of (60) there remain still points 1 and 2 as free. They can contract together giving a combined contraction

$$
1: 2 \cap 3: 4
$$

In fact the presence of point 4 considered alone as free does not stop the contractions of the time points 1,2 , and 3 which are:

$$
1: 2,1: 3,1: 2: 3 \text { and } 2: 3 \text {. }
$$

The above contractions (58)-(61) give the following energy terms:

$$
\begin{aligned}
& 1: 4 \rightarrow-\Delta E_{3}\left\langle V P^{2} V\right\rangle, \\
& 1: 2: 4 \rightarrow \Delta E_{1} \Delta E_{2}\left\langle V P^{3} V\right\rangle, \\
& 1: 3: 4 \rightarrow \Delta E_{2} \Delta E_{1}\left\langle V P^{3} V\right\rangle, \\
& 1: 2: 3: 4 \rightarrow-\left(\Delta E_{1}\right)^{3}\left\langle V P^{4} V\right\rangle, \\
& 2: 4 \rightarrow-\Delta E_{2}\left\langle V P V P^{2} V\right\rangle, \\
& 2: 3: 4 \rightarrow\left(\Delta E_{1}\right)^{2}\left\langle V P V P^{3} V\right\rangle, \\
& 3: 4 \rightarrow-\Delta E_{1}\left\langle V P V P V P^{2} V\right\rangle, \\
& 1: 2 \cap 3: 4 \rightarrow\left(\Delta E_{1}\right)^{2}\left\langle V P^{2} V P^{2} V\right\rangle .
\end{aligned}
$$

The presence of the free points 1,2, 3 before 4 yields the term

$$
1,2,3 \rightarrow\langle V P V P V P V P V\rangle
$$

given already in (45), but contractions listed in (62) provide us with the terms:

$$
\begin{aligned}
& 1: 2 \rightarrow-\left\langle V P^{2} V P V P V\right\rangle \Delta E_{1}, \\
& 1: 3 \rightarrow-\left\langle V P^{2} V P V\right\rangle \Delta E_{2}, \\
& 1: 2: 3 \rightarrow\left\langle V P^{3} V P V\right\rangle\left(\Delta E_{1}\right)^{2}, \\
& 2: 3 \rightarrow-\left\langle V P V P^{2} V P V\right\rangle \Delta E_{1} .
\end{aligned}
$$

The first contraction in (58) provides us with a factor having the perturbation energy $\Delta E_{3}$ which implies two Schrödinger terms, any of the remainder 11 contractions in (58)-(62) provide us with one Schrödinger perturbation term, so in total we obtain $2+11=13$ energy terms which-together with the term in (45) or (45a)-complete the calculation of 14 terms entering $\Delta E_{5}$.

\section{Comparison of the Born-Heisenberg-Jordan (BHJ) Perturbation Approach with That Given by Schrödinger}

The BHJ perturbation approach to a quantum system [19] was proposed almost simultaneously with that due to Schrödinger [3]. In a review of the quantum theory presented by Van der Waerden [20] the author considers the BHJ formalism as mathematically equivalent to the Schrödinger's method. This seems to be true only for the fragments of the $\mathrm{BHJ}$ calculation. In fact the first step done 
by $\mathrm{BHJ}$ - but absent in the Schrödinger case-is a conversion of the Hamiltonian $\hat{H}(\hat{p}, \hat{q})$ of a non-degenerate perturbed system into a diagonal matrix form

$$
\hat{H}(\hat{p}, \hat{q})=\hat{S} \hat{H}\left(\hat{p}_{0}, \hat{q}_{0}\right) \hat{S}^{-1}=\hat{W},
$$

so the commutation properties of $\hat{p}_{0}$ and $\hat{q}_{0}$ as well $\hat{p}$ and $\hat{q}$ remain unchanged. The next step-also absent in the Schrödinger theory-is to undertake a principal axes transformation of the Hermitian form given in (66) below into a sum of squares:

$$
\sum_{k, l} H(k l) x_{k} x_{l}^{*}=\sum_{m} W_{m} y_{m} y_{m}^{*} .
$$

The aim is to find the solution of the equation

$$
W x_{k}-\sum_{l} H(k l) x_{l}=0
$$

for some special $W=W_{n}$ with the aid of the substitutions

$$
\begin{gathered}
H(k l)=\delta_{k l} W_{l}^{(0)}+\lambda H^{(1)}(k l)+\lambda^{2} H^{(2)}(k l)+\cdots \\
W=W^{(0)}+\lambda W^{(1)}+\lambda^{2} W^{(2)}+\cdots
\end{gathered}
$$

and

$$
x_{k}=x_{k}^{(0)}+\lambda x_{k}^{(1)}+\lambda^{2} x_{k}^{(2)}+\cdots
$$

This leads to a sequence of results [19]:

$$
\begin{gathered}
W^{(1)}=H^{(1)}(n n), \\
W^{(2)}=H^{(2)}(n n)+\sum_{l}^{\prime} \frac{H_{l l}^{(1)} H_{l n}^{(1)}}{h v_{0}(n l)},
\end{gathered}
$$

and

$$
\begin{aligned}
W^{(3)}= & H^{(3)}(n n)+\sum_{l}^{\prime} \frac{H_{n l}^{(1)} H_{l n}^{(2)}+H_{n l}^{(2)} H_{n l}^{(1)}}{h v_{0}(n l)}+\sum_{l, k}^{\prime} \frac{H_{n l}^{(1)} H_{l k}^{(1)} H_{k n}^{(1)}}{h^{2} v_{0}(n l) v_{0}(k n)} \\
& -H_{n n}^{(1)} \sum_{l}^{\prime} \frac{H_{n l}^{(1)} H_{l n}^{(1)}}{h^{2} v_{0}^{2}(n l)}
\end{aligned}
$$

where $v_{0}(\alpha \beta)$ is the frequency of transition between the levels $\alpha$ and $\beta$.

The perturbation expansion of the matrix elements $H(k l)$ in (68) is absent in the Schrödinger perturbation theory, also expansion (70) is absent there. In effect only the term on the right of (71), the second term on the right of (72), and two last terms on the right of (73) - all containing the perturbed matrix elements of order one-are identical with the results obtained by Schrödinger.

\section{Discussion}

We find that the notion of time-very common in the everyday life and science, but not so necessary in mathematics-becomes very helpful in solving the Schrödinger perturbation problem for energy. In fact the corresponding energy series can be obtained from the combinatorics of the time-point contractions entering a circular time scale characteristic for the successive perturbation or- 
ders $N$.

We obtain here an example of the "action" of time as an ordering parameter which facilitates the calculations-in an agreement with the Leibniz idea of time as an arrangement parameter [21] [22].

The paper considers the solution of the Schrödinger differential eigenequation for energy being basic in the quantum theory. Usually to obtain such solution the well-known prescriptions concerning the differential equations have to be satisfied. But the Schrödinger's idea, and that of his followers, was to construct the eigenvalues of energy and perturbed eigenfunctions based on the ready solutions of a less complicated eigenequation. In fact such construction-making a systematic reference between the perturbed eigenequation and unperturbed one-became a very tedious task, especially for large perturbation orders $N$ which can be important for the accuracy of the solution.

The advantage of the applied time scale is that the formulae for the terms of the perturbation energy can be calculated almost immediately on the basis of an analysis of the results provided by the scale.

\section{Conflicts of Interest}

The author declares no conflicts of interest regarding the publication of this paper.

\section{References}

[1] Schrödinger, E. (1926) Quantisierung als Eigenwertproblem (erste Mitteilung). Annalen der Physik, 79, 361-376. https://doi.org/10.1002/andp.19263840404

[2] Schrödinger, E. (1926) Quantisierung als Eigenwertproblem II. Annalen der Physik, 79, 489-527. https://doi.org/10.1002/andp.19263840602

[3] Schrödinger, E. (1926) Quantisierung als Eigenwertproblem Ill. Annalen der Physik, 80, 437-490. https://doi.org/10.1002/andp.19263851302

[4] Schrödinger, E. (1926) Quantisierung als Eigenwertproblem (vierte Mitteilung). Annalen der Physik, 81, 109-139. https://doi.org/10.1002/andp.19263861802

[5] Seitz, F. (1940) Modern Theory of Solids. McGraw-Hill, New York.

[6] Seitz, F. and Turnbull, D. (Eds.) (1955) E. P. Wigner in Solid State Physics Vol. 1. Academic Press, New York.

[7] Slater, J.C. (1960) Quantum Theory of the Atomic Structure. Vol. 1 and 2, McGraw-Hill, New York.

[8] Schiff, L.I. (1968) Quantum Mechanics. 3rd Edition, McGraw-Hill, New York.

[9] Huby, R. (1961) Formulae for Non-Degenerate Rayleigh-Schrödinger Perturbation Theory in Any Order. Proceedings of the Physical Society (London), 78, 529.

[10] Tong, B.Y. (1962) On Huby's Rules for Non-Degenerate Rayleigh-Schrödinger Perturbation Theory in any Order. Proceedings of the Physical Society (London), 80, 1101.

[11] Feynman, R.P. (1966) The Development of the Space-Time View of Quantum Electrodynamics. Science, 153, 699-708.

[12] Mattuck, R.D. (1976) A Guide to Feynman Diagrams in a Many-Body Problem. 2nd Edition, McGraw-Hill, New York. 
[13] Olszewski, S. (1991) Time Scale and Its Application in Perturbation Theory. Zeitschrift fuer Naturforschung, 46A, 313.

[14] Olszewski, S. and Kwiatkowski, T. (1998) A Topological Approach to Evaluation of Non-Degenerate Schrödinger Perturbation Energy Based on a Circular Scale of Time. Computers in Chemistry, 22, 445-461. https://doi.org/10.1016/S0097-8485(98)00023-0

[15] Olszewski, S. (2003) Two Pathways of the Time Parameter Characteristic for the Perturbation Problem in Quantum Chemistry. Trends in Physical Chemistry, 9, 69.

[16] Olszewski, S. (2013) A Look on the Scale of Time Useful in the Non-Relativistic Quantum Mechanics. Quantum Matter, 2, 481.

[17] Olszewski, S. (2004) Kołowa skala czasu z punktu widzenia mechaniki kwantowej w ujęciu Schrödingera. Studia Philosophiae Christianae, 40, 57-81.

[18] Olszewski, S. (2017) Circular Scale of Time and Energy of a Quantum State Calculated from the Schrödinger Perturbation Theory. Journal of Modern Physics, 5, 1502.

[19] Born, M., Heisenberg, W. and Jordan, P. (1926) Zur Quantenmechanik. II. Zeitschrift fuer Physik, 35, 557-615. https://doi.org/10.1007/BF01379806

[20] Van der Waerden, B.L. (1968) Sources of Quantum Mechanics. Dover Publications, New York.

[21] Leibniz, G.W. (1924) Hauptschriften zur Grundlagen der Philosophie. Vol. 1, Leipzig.

[22] Rescher, N. (2013) On Leibniz. University of Pittsburgh Press, Pittsburgh. https://doi.org/10.2307/j.ctt7zw8g2 